\title{
Identification of context-dependent expression quantitative trait loci in whole blood
}

Citation for published version (APA):

Zhernakova, D. V., Deelen, P., Vermaat, M., van Iterson, M., van Galen, M., Arindrarto, W., van 't Hof, P., Mei, H., van Dijk, F., Westra, H-J., Bonder, M. J., van Rooij, J., Verkerk, M., Jhamai, P. M., Moed, M., Kielbasa, S. M., Bot, J., Nooren, I., Pool, R., ... Franke, L. (2017). Identification of context-dependent expression quantitative trait loci in whole blood. Nature Genetics, 49(1), 139-145.

https://doi.org/10.1038/ng.3737

Document status and date:

Published: 01/01/2017

DOI:

10.1038/ng.3737

Document Version:

Publisher's PDF, also known as Version of record

Document license:

Taverne

Please check the document version of this publication:

- A submitted manuscript is the version of the article upon submission and before peer-review. There can be important differences between the submitted version and the official published version of record.

People interested in the research are advised to contact the author for the final version of the publication, or visit the DOI to the publisher's website.

- The final author version and the galley proof are versions of the publication after peer review.

- The final published version features the final layout of the paper including the volume, issue and page numbers.

Link to publication

\footnotetext{
General rights rights.

- You may freely distribute the URL identifying the publication in the public portal. please follow below link for the End User Agreement:

www.umlib.nl/taverne-license

Take down policy

If you believe that this document breaches copyright please contact us at:

repository@maastrichtuniversity.nl

providing details and we will investigate your claim.
}

Copyright and moral rights for the publications made accessible in the public portal are retained by the authors and/or other copyright owners and it is a condition of accessing publications that users recognise and abide by the legal requirements associated with these

- Users may download and print one copy of any publication from the public portal for the purpose of private study or research.

- You may not further distribute the material or use it for any profit-making activity or commercial gain

If the publication is distributed under the terms of Article $25 \mathrm{fa}$ of the Dutch Copyright Act, indicated by the "Taverne" license above, 


\title{
Identification of context-dependent expression quantitative trait loci in whole blood
}

\author{
Daria V Zhernakova ${ }^{1,20}$, Patrick Deelen ${ }^{1,2,20}$, Martijn Vermaat ${ }^{3,20}$, Maarten van Iterson ${ }^{4,20}$, Michiel van Galen ${ }^{3}$, \\ Wibowo Arindrarto ${ }^{5}$, Peter van 't Hof ${ }^{5}$, Hailiang Mei ${ }^{5}$, Freerk van Dijk ${ }^{1,2}$, Harm-Jan Westra ${ }^{6-8}$, Marc Jan Bonder ${ }^{1}$, \\ Jeroen van Rooij ${ }^{9}$, Marijn Verkerk ${ }^{9}$, P Mila Jhamai ${ }^{9}$, Matthijs Moed ${ }^{4}$, Szymon M Kielbasa ${ }^{4}$, Jan Bot ${ }^{10}$, Irene Nooren ${ }^{10}$, \\ René Pool ${ }^{11}$, Jenny van Dongen ${ }^{11}$, Jouke J Hottenga ${ }^{11}$, Coen D A Stehouwer ${ }^{12,13}$, Carla J H van der Kallen ${ }^{12,13}$, \\ Casper G Schalkwijk ${ }^{12,13}$, Alexandra Zhernakova ${ }^{1}$, Yang Li ${ }^{1}$, Ettje F Tigchelaar ${ }^{1}$, Niek de Klein ${ }^{1}$, Marian Beekman ${ }^{4}$, \\ Joris Deelen ${ }^{4}$, Diana van Heemst ${ }^{14}$, Leonard H van den Berg ${ }^{15}$, Albert Hofman ${ }^{16}$, André G Uitterlinden ${ }^{9}$, \\ Marleen M J van Greevenbroek ${ }^{12,13}$, Jan H Veldink ${ }^{15}$, Dorret I Boomsma ${ }^{11}$, Cornelia M van Duijn ${ }^{17}$, \\ Cisca Wijmenga ${ }^{1}$, P Eline Slagboom ${ }^{4}$, Morris A Swertz ${ }^{1,2}$, Aaron Isaacs ${ }^{13,17,18}$, Joyce B J van Meurs ${ }^{9}$, \\ Rick Jansen ${ }^{19}$, Bastiaan T Heijmans ${ }^{4,21}$, Peter A C't Hoen ${ }^{3,21} \&$ Lude Franke ${ }^{1,21}$
}

\begin{abstract}
Genetic risk factors often localize to noncoding regions of the genome with unknown effects on disease etiology ${ }^{1,2}$. Expression quantitative trait loci (eQTLs) help to explain the regulatory mechanisms underlying these genetic associations ${ }^{3-6}$. Knowledge of the context that determines the nature and strength of eQTLs may help identify cell types relevant to pathophysiology and the regulatory networks underlying disease ${ }^{7-17}$. Here we generated peripheral blood RNA-seq data from 2,116 unrelated individuals and systematically identified context-dependent eQTLs using a hypothesis-free strategy that does not require previous knowledge of the identity of the modifiers. Of the 23,060 significant cis-regulated genes (false discovery rate $(F D R) \leq 0.05), 2,743(12 \%)$ showed context-dependent eQTL effects. The majority of these effects were influenced by cell type composition. A set of 145 cis-eQTLs depended on type I interferon signaling. Others were modulated by specific transcription factors binding to the eQTL SNPs.
\end{abstract}

We created the Biobank-Based Integrative Omics Study (BIOS) data set by sequencing whole peripheral blood mRNA in 2,116 healthy adults from four Dutch cohorts ${ }^{18-21}$ (Supplementary Table 1 and
Supplementary Note; EGAD00001001623). We quantified gene and exon expression, as well as exon ratios (the proportion of expression of an exon relative to the total expression of all exons of a gene) and poly $(\mathrm{A})$ ratios (the ratio of expression in upstream and downstream parts of 3' UTRs separated by annotated polyadenylation (poly(A)) sites), and performed cis-eQTL mapping for all of these (Supplementary Note). We detected cis-eQTL effects for $66 \%$ of the protein-coding genes and $19 \%$ of the noncoding genes tested. In total, we identified eQTL effects for 23,060 different genes (FDR $\leq$ 0.05 ). These eQTLs replicated well in earlier microarray-based data sets from blood samples ${ }^{22}$ and an RNA-seq data set based on lymphoblastoid cell lines (LCLs) ${ }^{23}$ (Supplementary Note), but they also substantially extended the list of genes that are known to be under genetic regulation (replication results are given in the Supplementary Note and Supplementary Table 2). In addition to detecting gene-level eQTLs, we identified 21,888 different genes with one or more exonlevel QTL effects and 9,777 and 2,322 genes where SNPs affected the inclusion rate of exons and the usage of poly(A) sites, respectively (Supplementary Table 3). All QTLs can be found using our QTL browser (http://genenetwork.nl/biosqtlbrowser). Multiple unlinked SNPs in the same locus may independently influence expression or

\footnotetext{
1 University of Groningen, University Medical Center Groningen, Genomics Coordination Center, Groningen, the Netherlands. 2University of Groningen, University Medical Center Groningen, Department of Genetics, Groningen, the Netherlands. ${ }^{3}$ Department of Human Genetics, Leiden University Medical Center, Leiden, the Netherlands. ${ }^{4}$ Molecular Epidemiology Section, Department of Medical Statistics and Bioinformatics, Leiden University Medical Center, Leiden, the Netherlands. ${ }^{5}$ Sequence Analysis Support Core, Leiden University Medical Center, Leiden, the Netherlands. ${ }^{6}$ Divisions of Genetics and Rheumatology, Department of Medicine, Brigham and Women's Hospital and Harvard Medical School, Boston, Massachusetts, USA. 7 Partners Center for Personalized Genetic Medicine, Boston, Massachusetts, USA. ${ }^{8}$ Program in Medical and Population Genetics, Broad Institute of MIT and Harvard, Cambridge, Massachusetts, USA. ${ }^{9}$ Department of Internal Medicine, ErasmusMC, Rotterdam, the Netherlands. ${ }^{10}$ SURFsara, Amsterdam, the Netherlands. ${ }^{11}$ Department of Biological Psychology, Vrije Universiteit Amsterdam, Neuroscience Campus Amsterdam, Amsterdam, the Netherlands. ${ }^{12}$ Department of Internal Medicine, Maastricht University Medical Center, Maastricht, the Netherlands. ${ }^{13}$ School for Cardiovascular Diseases (CARIM), Maastricht University Medical Center, Maastricht, the Netherlands. ${ }^{14}$ Department of Gerontology and Geriatrics, Leiden University Medical Center, Leiden, the Netherlands. ${ }^{15}$ Department of Neurology, Brain Center Rudolf Magnus, University Medical Center Utrecht, Utrecht, the Netherlands. ${ }^{16}$ Department of Epidemiology, ErasmusMC, Rotterdam, the Netherlands. ${ }^{17}$ Genetic Epidemiology Unit, Department of Epidemiology, ErasmusMC, Rotterdam, the Netherlands. ${ }^{18}$ Maastricht Centre for Systems Biology (MaCSBio), Maastricht University, Maastricht, the Netherlands. ${ }^{19}$ Department of Psychiatry, VU University Medical Center, Neuroscience Campus Amsterdam, Amsterdam, the Netherlands. ${ }^{20}$ These authors contributed equally to this work. ${ }^{21}$ These authors jointly directed this work. Correspondence should be addressed to L.F. (lude@ludesign.nl), P.A.C.'t.H. (p.a.c._t_hoen@lumc.nl) or B.T.H. (b.t.heijmans@lumc.nl).
} 
a

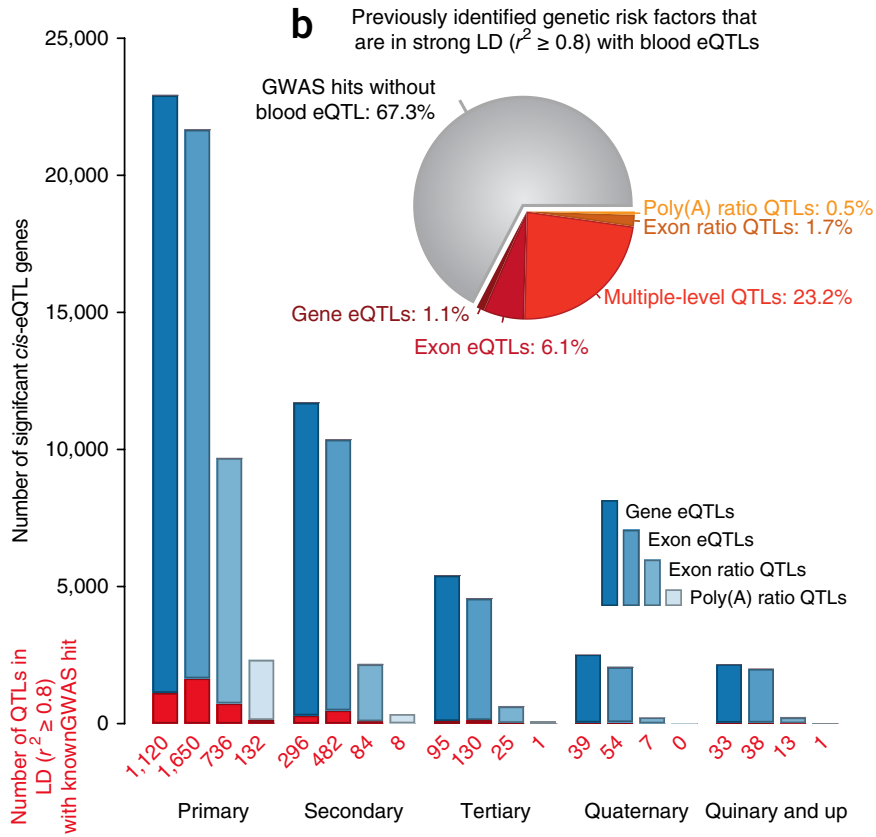

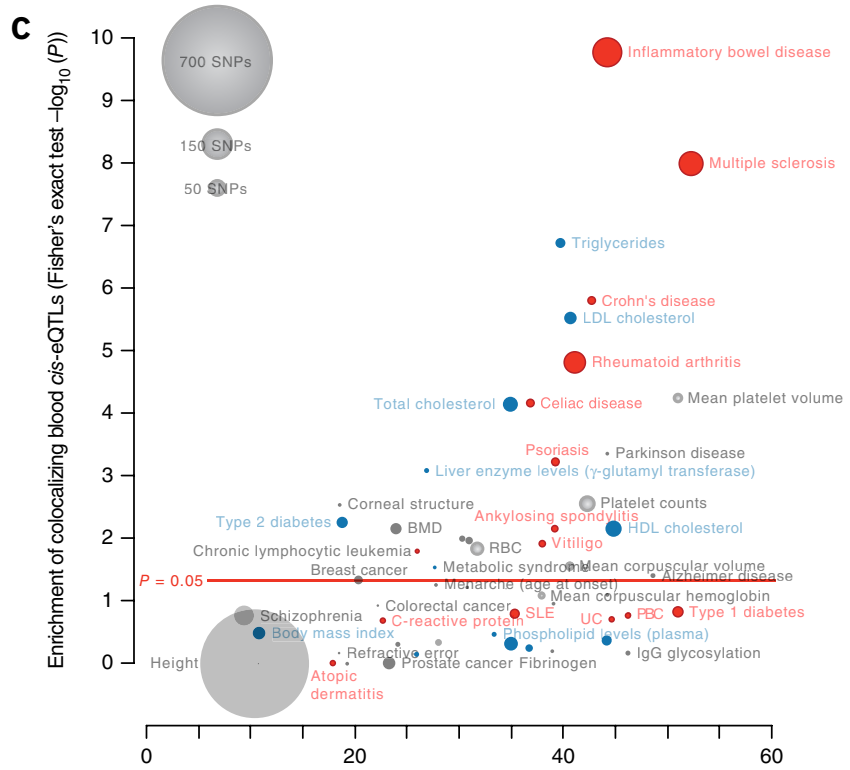

Proportion of trait-associated SNPs in strong LD $\left(r^{2} \geq 0.8\right)$ with eQTL SNP $(\%)$

Figure 1 Over 20,000 genes are regulated by cis-eQTLs overlapping with 33\% of the entries in the NHGRI GWAS catalog. (a) Number of cis-regulated genes having one, two, three, four, and five or more independent eQTL effects (FDR $\leq 0.05$ ). The number of eQTLs overlapping with SNPs in the GWAS catalog $\left(r^{2} \geq 0.8\right)$ appears in red. (b) Distribution of GWAS catalog variants over the different types of QTLs. Of the GWAS catalog SNPs, $8 \%$ affected exon-level QTLs or poly(A) ratio QTLs but did not affect overall gene expression levels. (c) Risk loci for autoimmune disorders and traits related to blood showed higher colocalization with eQTLs than those for anthropometric traits and diseases without an immune or hematological component. The $x$ axis shows the percentage of GWAS hits colocalizing with eQTLs, and the $y$ axis shows enrichment of overlap (calculated at the gene level), using genes associated with height as a conservative background.

mRNA processing of the same gene ${ }^{24}$. This was observed for more than half of the cis-regulated genes (Fig. 1a and Supplementary Fig. 1).

The gene-level cis-eQTL SNPs were strongly enriched for DNase I footprints, various histone marks and binding sites for multiple transcription factors ${ }^{25}$ (Supplementary Table 4 and Supplementary Note), suggesting the likely detection of causal regulatory variants. Moreover, top eQTL SNPs were significantly enriched for general enhancers and ones specific to blood cell types (taken from ref. 26), but not for enhancers specific to non-blood tissues (Supplementary Table 5). Evidence for the functionality of exon ratio and poly(A) ratio QTLs in mRNA splicing and polyadenylation, respectively, is also presented in the Supplementary Note.

One-third (2,064, or $32.7 \%)$ of previously established genetic risk factors for disease or complex traits (derived from the National Human Genome Research Institute (NHGRI) genome-wide association study (GWAS) catalog and a set of reported Immunochip associations, $P \leq 5 \times 10^{-8}$; Supplementary Table 6) were in strong linkage disequilibrium (LD; $r^{2} \geq 0.8$ ) with a top eQTL SNP (Fig. 1b and Supplementary Table 7). As expected, eQTL effects were predominantly found for SNPs associated with hematological, lipid or immune-related traits. We observed highly significant enrichment of colocalization of eQTL and GWAS SNPs $\left(r^{2} \geq 0.8\right)$ for many immune disorders in comparison to the $10 \%$ overlap found for height, which we considered to represent a conservative background level (inflammatory bowel disease (IBD), $1.6 \times 10^{-10}$; multiple sclerosis, $9.8 \times 10^{-9}$; rheumatoid arthritis, $\left.1.5 \times 10^{-5}\right)$ (Fig. 1c and Supplementary Note). This indicates that our blood cis-eQTLs are highly informative for diseases such as IBD, multiple sclerosis and rheumatoid arthritis.

Effect sizes for eQTLs often depend on the cell type or tissue under investigation ${ }^{8-11,27,28}$ and may be modified by external and environmental factors ${ }^{14-17,29,30}$. We developed a hypothesis-free strategy to identify which of the observed eQTLs were dependent on intrinsic or extrinsic factors (context-dependent eQTLs) (Fig. 2a,b, Online Methods and Supplementary Fig. 2). Instead of using known factors, such as the percentage of neutrophils in blood in a gene-byenvironment interaction model ${ }^{13}$, we used the expression levels of other genes as interaction factors. We call these genes 'proxy genes', as they may reflect the abundance of a cell type or the activity of signaling pathways.

We identified 10 modules of in total 1,842 eQTLs independently affected by 10 largely uncorrelated proxy genes (Fig. $2 \mathrm{c}$ and Supplementary Table 8). eQTLs with context-dependent effects can be obtained from our BIOS eQTL browser. An example is shown in Figure 2b, where we found an eQTL effect of SNP rs1981760 (a SNP associated with leprosy susceptibility) on NOD2 expression. Expression of the first top proxy gene, STX3, had a significant interaction with this eQTL. Samples with very low expression of STX3 showed only a very weak eQTL effect on NOD2, whereas samples with very high STX3 expression showed a stronger eQTL effect. Further analysis demonstrated that STX3 expression was strongly correlated (Pearson $r=0.74$ ) with the percentage of neutrophils in the blood, indicating that STX3 is a proxy for neutrophil levels in blood.

It can be challenging to understand what the proxy genes represent. We first assessed whether they are markers for specific cell types and correlated them with blood cell counts measured in our samples (for neutrophils, lymphocytes, eosinophils, basophils and monocytes) and baseline gene expression levels in purified blood cells from the BLUEPRINT consortium ${ }^{31}$ (Fig. 2c and Supplementary Fig. 3). Eight of the ten proxy genes likely represent the levels of specific cell types in blood (Supplementary Note). Analysis of eQTL gene expression in BLUEPRINT data (Supplementary Fig. 4a) and eQTL interactions with measured blood cell counts confirmed 
a

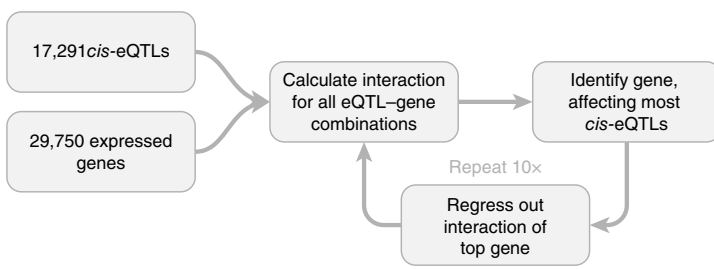

b

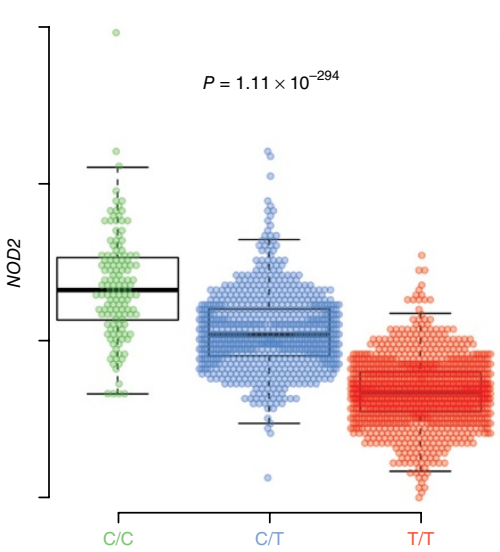

Leprosy risk SNP rs 1981760
NOD2 eQTL in 106 samples with STX3 gene expression levels

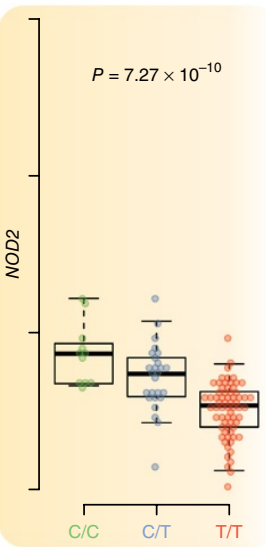

rs1981760
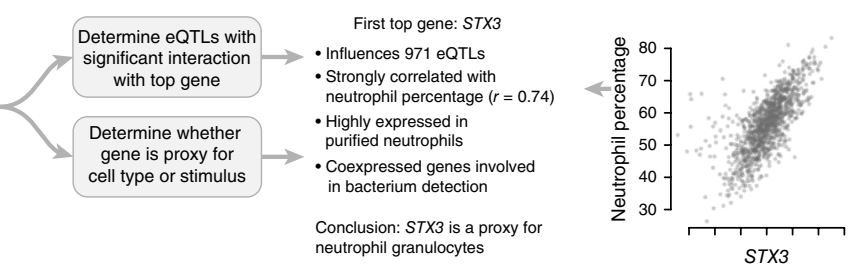

STX3
NOD2 eQTL in 106 samples with high STX3 gene expression levels
NOD2 eQTL interaction analysis,
STX3 interacts with rs 1981760

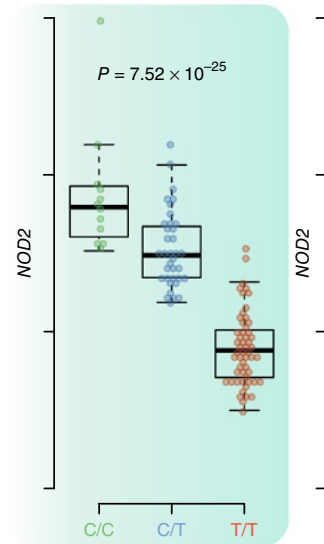

rs1981760

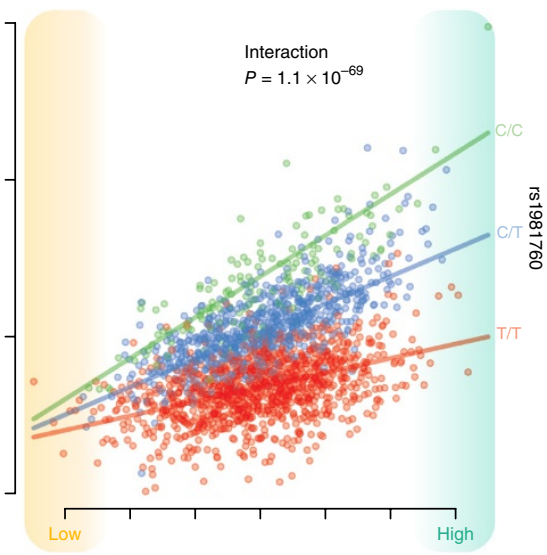

STX3

C

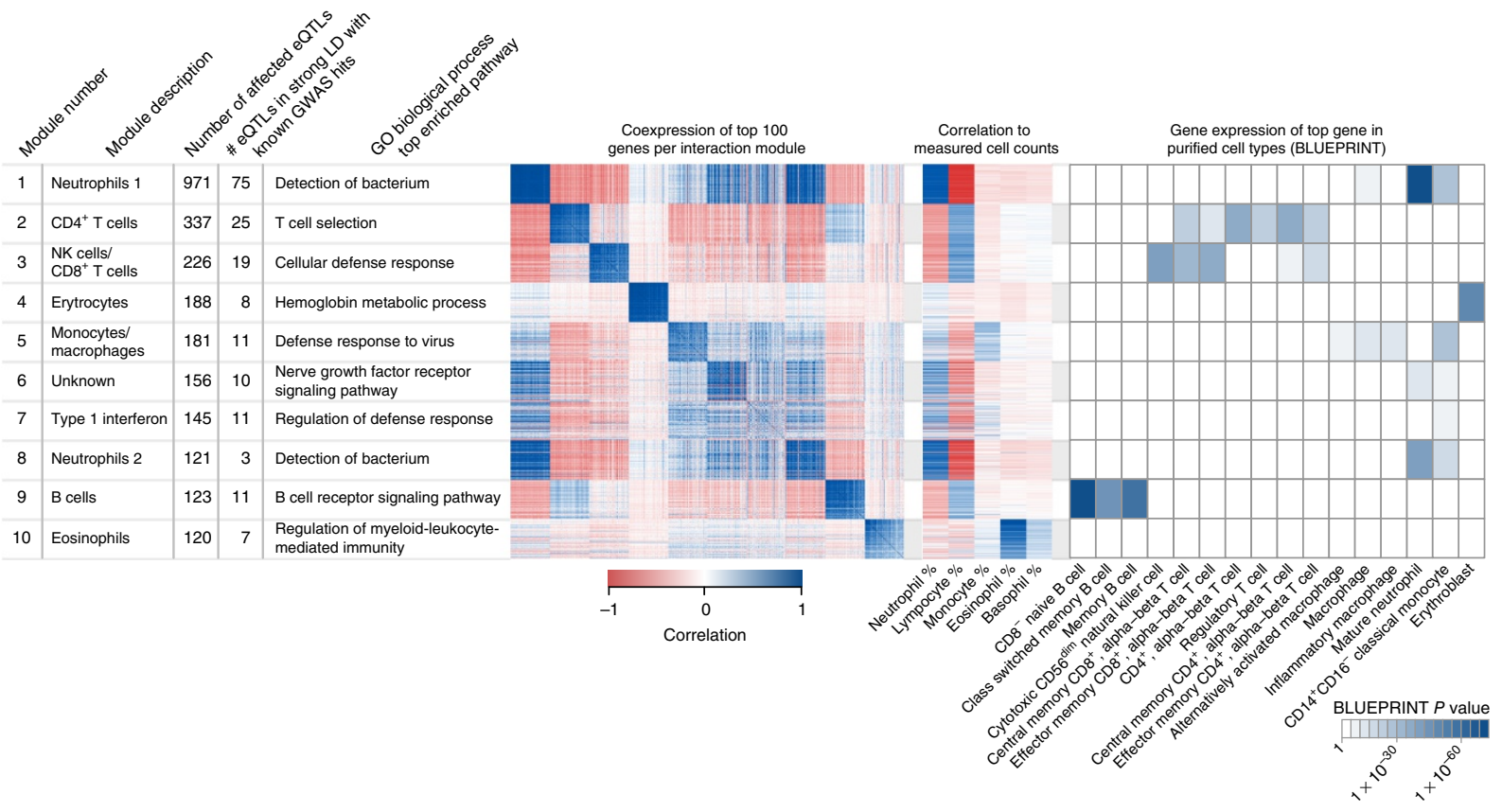

Figure 2 Identification of the strongest modifiers of eQTL effects. (a) Overview of the method used to annotate eQTLs as context dependent: each highly expressed gene (with at least one read in all samples) was tested for its ability to modify each of the 17,291 eQTLs affecting highly expressed genes. For each of the identified proxy genes, we determined the overall strength of the interaction effects with all eQTLs. We selected the strongest proxy gene and regressed its effects from the data. We did this for ten iterations, allowing the identification of ten independent proxy genes that affect the strength of eQTLs. (b) An example of a context-dependent eQTL effect is rs1981760, a strong eQTL for the NOD2 gene. This SNP is in strong LD $\left(r^{2}=0.99\right)$ with rs9302752, a variant associated with leprosy susceptibility. The leprosy risk allele (T) results in decreased expression of NOD2. In samples with low STX3A expression, only a weak eQTL effect is observed, whereas in samples with high STX3A expression a strong eQTL effect is observed. In accordance with these findings, using the STX3A gene as a covariate in an interaction model shows a very strong interaction effect. STX $3 A$ is the first proxy gene we identified, and its expression correlates strongly with neutrophil percentage (Pearson $r=0.72$ ). Gene enrichment analysis of STX3A and other genes exhibiting similar interaction patterns shows involvement in antibacterial response. Furthermore, individuals carrying the leprosy risk allele have significantly weaker NOD2 upregulation in neutrophils than non-carriers. This is in line with earlier reports showing this eQTL to be stronger in FACS-sorted neutrophils than in monocytes ${ }^{27}$. Box plots show the median, the first and third quartiles, and 1.5 times the interquartile range. (c) We annotated each of our 10 proxy genes using the top 100 proxy genes from each module with similar effects and showed that, as expected, these top 100 genes are strongly correlated in each module. These sets of the top 100 proxies were used for gene function enrichment analysis (for full results, see Supplementary Table 12) and are correlated to known cell proportions. We used BLUEPRINT expression data for sorted populations of blood cells to validate cell-type-specific expression in each module; $n=2,116$ individuals were used in the analysis. 
the cell-type-dependent effects of neutrophils and eosinophils (Supplementary Fig. 5 and Supplementary Tables 9 and 10), but our unbiased analysis also identified effects for cell types for which actual cell counts were not available (erythroblasts, CD4 ${ }^{+}$
T cells and natural killer (NK) cells/CD8 ${ }^{+} \mathrm{T}$ cells). Replication of our cell-type-dependent eQTLs in eQTL data sets from purified cell types supported these observations (Supplementary Fig. 4b,c and Supplementary Table 11). a

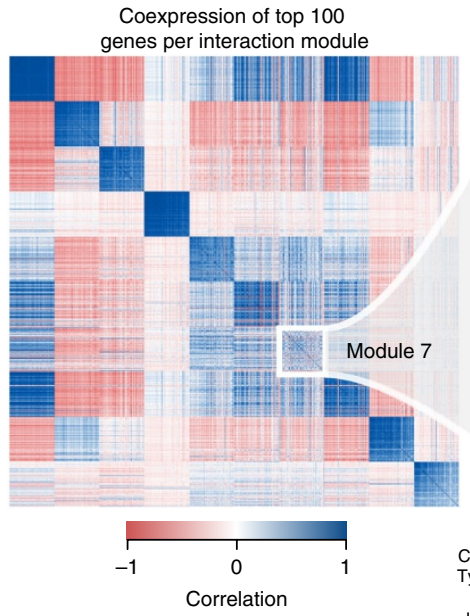

Module 7, top 100 genes

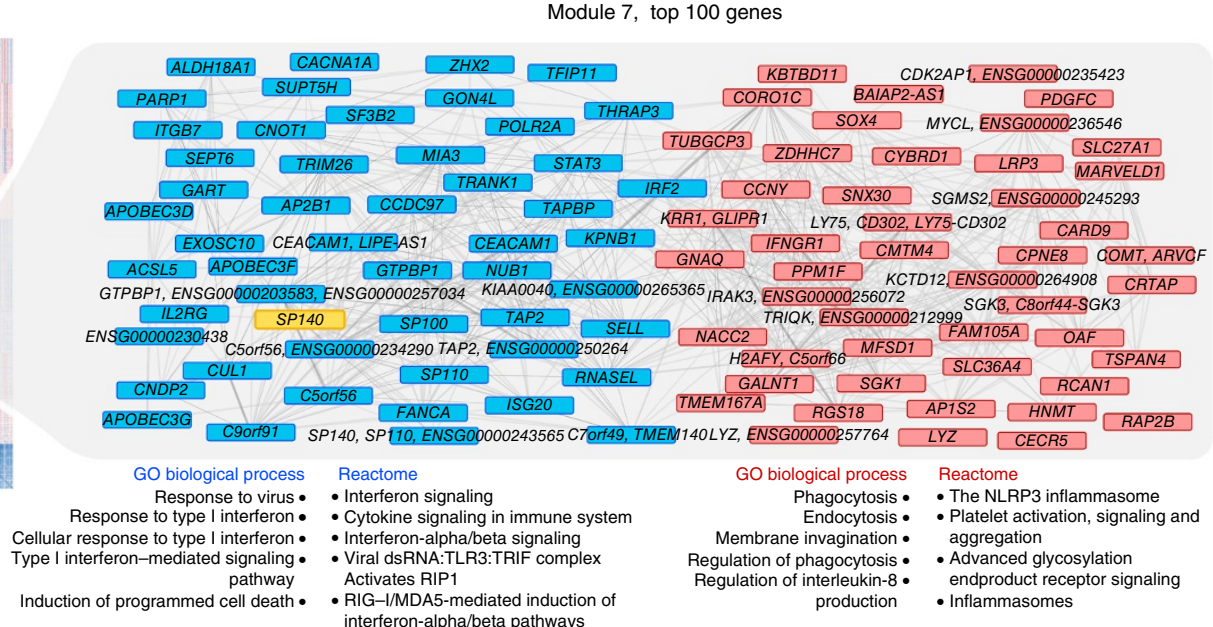

b

SP140 Module 7 top covariate gene Gene Positive correlation to SP140 Gene Negative correlation to SP140

Overlap with ChIP-seq

STAT1 6 h after IFN- $\alpha$

STAT2 6 h after IFN- $\alpha$

STAT3

eQTLs with significant interaction with module 7 top covariate gene SP140

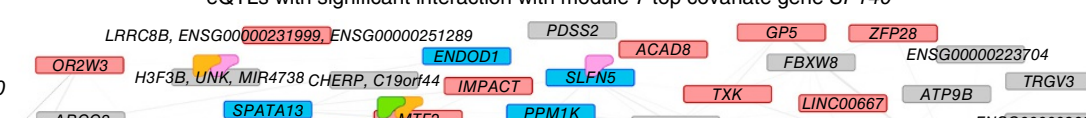

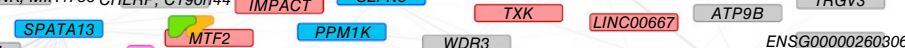

$$
\text { IFN- } \alpha
$$

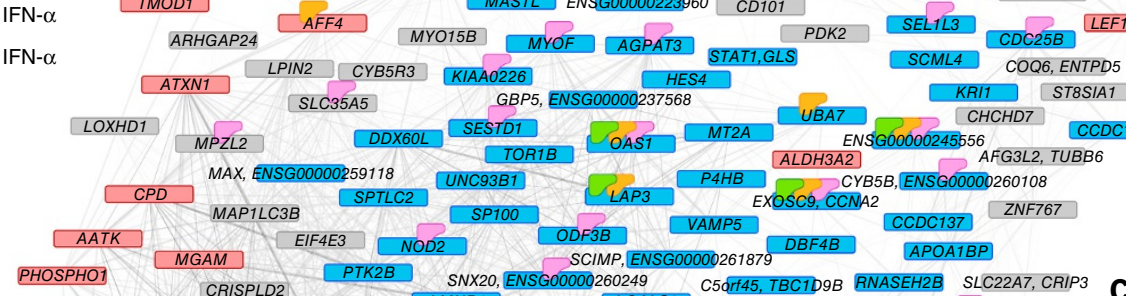

ARHGAP24 AFF4 MYO15B MYOFTL ENSG00000223960 CD101 \begin{tabular}{llll} 
PHOSPHO1 & \multicolumn{1}{c}{ CRISPLD2 } & PTK2B & SNX20, \\
NKKX3-1 & PGM2 & RBM23 & LMNB1 \\
\hline TOR1A
\end{tabular} BAIAP2, AATK CREB5 SELL EDEM2 RAB3D, ENSG00000267174, ENSG00000267576 FAM212B
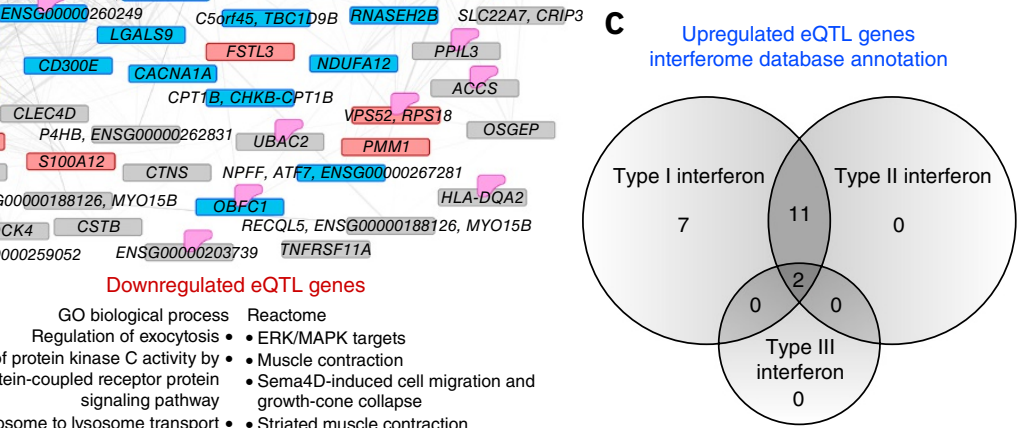

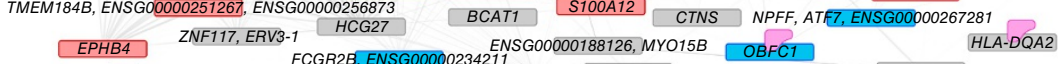

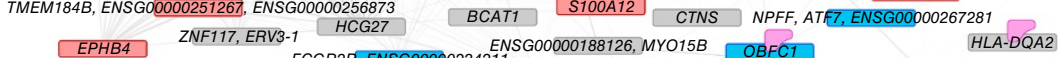

S100A12 CTS G00000262831 UBAC2 PMM1 FCGR2B, ENSG00000234211 ADCK4 CSTB RECQL5, ENSG00000188126, MYO15B Upregulated eQTL genes GO biological process Reactome Response to type I interferon $\bullet$ - Interferon signaling Type I interferon production $\bullet$ Interferon-alpha/beta signaling Cellular response to type I interferon $\bullet$ - Cytokine signaling in immune system Type I interferon-mediated signaling • • Regulation of interferon-alpha signaling Response to cytokine stimulus pathway TRAF6-mediated IRF7 activation

$$
\begin{aligned}
& \begin{array}{ccc}
\text { ADCK4 } & \text { CSTB } & \text { RECQL5, ENSG0000 } \\
\text { ENSG00000259052 ENSG00000203739 TNFRSF11A }
\end{array} \\
& \text { Downregulated eQTL genes } \\
& \text { GO biological process Reactome } \\
& \begin{array}{l}
\text { Regulation of exocytosis } \bullet \text { ERK/MAPK targets } \\
\text { rotein kinase } C \text { activity by }- \text { Muscle contraction }
\end{array} \\
& \text { A tivation of protein kinase } C \text { activity by } \bullet \text { Muscle contraction } \\
& \text { G-protein-coupled receptor protein - Sema4D-induced cell migration an } \\
& \text { signaling pathway growth-cone collapse } \\
& \text { Endosome to lysosome transport • - Striated muscle contraction } \\
& \text { Regulation of vesicle-mediated transport } \bullet \cdot \text { Creation of C4 and C2 activators }
\end{aligned}
$$

Figure 3 eQTLs modified by type I interferon signaling. (a) Expression-based clustering of genes positively correlated (blue) and negatively correlated (red) with the proxy gene SP140. Enrichment analysis of these two clusters showed distinct biology: the upregulated genes are enriched for type I interferon response and response to viruses, whereas the downregulated genes indicate an antibacterial inflammatory response. Type I interferon signaling is activated in a viral response, and type II interferon signaling is activated upon bacterial response ${ }^{37}$. The positively correlated genes are enriched for genes upregulated upon rhinovirus stimulation ${ }^{15}$ (Fisher's exact $P=1.14 \times 10^{-9}$ ), in line with their involvement in the type I interferon response. In contrast, the negatively correlated genes are enriched for genes upregulated upon stimulation with lipopolysaccharide (LPS) (Fisher's exact $P=0.02$ ) and interferon- $\gamma\left(\text { Fisher's exact } P=8.72 \times 10^{-4}\right)^{14}$, supporting the antibacterial function of these genes. (b) The eQTLs affected by $S P 140$ expression can also be divided into genes positively and negatively correlated with $S P 140$ expression. The significantly positively correlated eQTL genes are also enriched for type I interferon response, whereas the negatively correlated eQTL genes do not show strong enrichment for biological functions. Genes bound by STAT transcription factors, as identified in ENCODE ChIP-seq data from LCLs, are labeled. Both type I and type II interferon signaling result in binding of heterodimers of the STAT1 transcription factor. Uniquely in type I interferon response STAT1 forms a complex with STAT2 and IRF9, resulting in the activation of viral response genes. STAT3 activation is also unique to the type I interferon response, resulting in the downregulation of inflammatory pathways ${ }^{38}$. The eQTLs were enriched for binding sites for STAT1 $\left(P=4.82 \times 10^{-4}\right)$, STAT2 $\left(P=3.12 \times 10^{-4}\right)$ and STAT3 $(P=4.72 \times$ $10^{-5}$ ) (based on ENCODE ChIP-seq experiments) (Supplementary Table 13). Motif enrichment analysis ${ }^{39}$ on the 25-bp regions flanking the eQTL SNPs confirmed the enrichment of STAT-binding motifs (Wilcoxon rank-sum $P=9.61 \times 10^{-5}$ ). (c) Interferome database annotation of the upregulated eQTL genes confirms their role in type I (and not type II or III) interferon signaling; $n=2,116$ individuals were used in all eQTL analyses. 
a



b

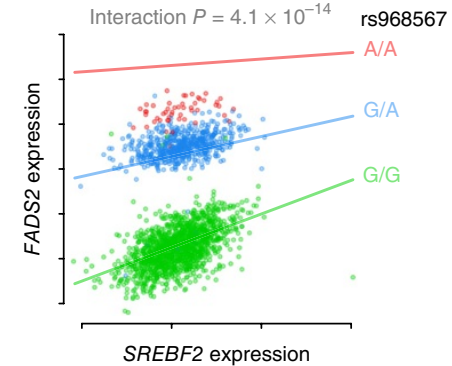

C

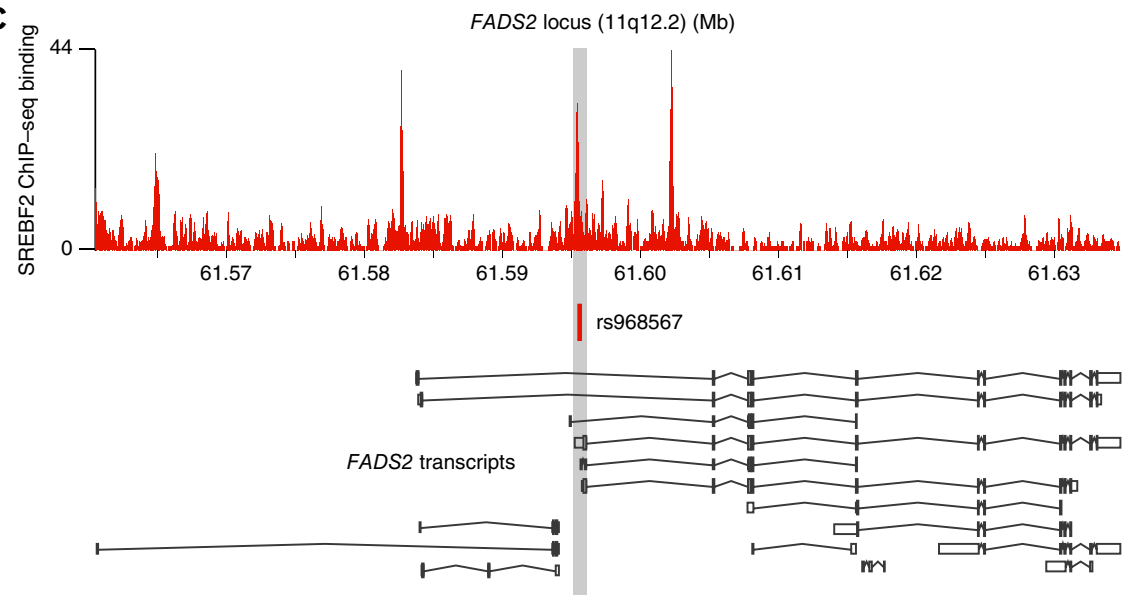

Figure 4 FADS2 eQTL modulated by SREBF2 expression. (a) The eQTL SNP rs968567 is located in a SREBF2-binding site in the FADS2 promoter. (b) The eQTL is modulated by SREBF2 expression and is stronger in samples with low SREBF2 expression. The nominal $P$ value for the interaction effect is given; $n=2,116$ individuals were used in the eQTL analysis. (c) rs968567 is located in an ENCODE ChIP-seq peak of SREBF2 binding.

Cell-type-specific eQTL genes were enriched in cell-typespecific signaling pathways (Fig. 2c and Supplementary Table 12). For example, the genes for which cis-eQTL effects were particularly strong in erythroblasts (represented by proxy gene TSPAN5) are enriched for erythrocyte-specific functions. They were also enriched in binding sites for transcription factors involved in erythrocyte development, on the basis of Encyclopedia of DNA Elements (ENCODE) ChIP-seq data (GATA1, TAL1, GATA2 and MAFK, each with an enrichment $P$ value $\left.\leq 1 \times 10^{-5}\right)^{32-34}$. A well-established cis-eQTL for SMIM1, an erythrocyte-specific gene encoding a protein that determines the Vel blood group 4 , was contained in the set of eQTLs affected by TSPAN5 expression. For eQTLs affected by other proxy genes, we also identified specific transcription factors with established functions in the corresponding cell types (Supplementary Table 13).

In Supplementary Figure 6 and the Supplementary Note, we show examples of how eQTLs can be used to gain insights into five autoimmune disorders. Clustering of the eQTL genes based on coexpression identified sets of genes hinting at specific cell types and biological functions. For IBD, for instance, the clustering identified a T cell cluster and a neutrophil cluster. Adding the cell-type-dependent eQTLs further corroborated the cell type annotations of the clusters. In total, we found 138 context-dependent eQTLs for GWAS-identified variants (Supplementary Table 14).

The identified interaction modules are not restricted to cell-typespecific effects. One of the proxy genes, SP140, is not a proxy for cell type but is a proxy for type I interferon response, as demonstrated by pathway enrichment of genes that correlated positively with SP140 expression levels (Supplementary Note). Genes that correlated negatively with $S P 140$ expression are involved in antibacterial response and inflammation (Fig. 3a). Likewise, the affected eQTL genes can be divided into two groups: those positively and those negatively correlated with SP140 expression (Fig. 3b). Gene annotations from the interferome database ${ }^{35}$ confirmed that the upregulated eQTL genes are indicative of type I, but not of type II, interferon response (Fig. 3c). In support of the modifying effects of viral cues on this set of eQTLs, eQTL genes that were recently reported as rhinovirus response QTLs ${ }^{15}$ typically demonstrated higher SP140 interaction effects than other eQTL genes (Wilcoxon $P=0.02$ ).

Each of the ten aforementioned proxy genes demonstrated effects on many $(>120)$ eQTLs. However, some other factors may also exist that affect more limited numbers of eQTLs. To identify these factors, we first corrected the expression data for the ten proxy genes and their eQTL interaction effects and then ascertained for each gene-level eQTL whether the size of the eQTL effect was significantly dependent on the expression of any other gene. This analysis resulted in the identification of an additional set of 901 context-dependent eQTLs $(F D R \leq 0.05)$ (Supplementary Table 15). Of these eQTL interactions, 113 could also be detected in Geuvadis LCLs (FDR $\leq 0.05 ; 94 \%$ with the same direction of interaction) (Supplementary Table 16). These LCLs are homogeneous cell populations, so any interaction effect that replicates is unlikely to be due to cell-type-specific eQTL effects but rather reflects external stimulation or activation of core biological processes. A few of these context-dependent eQTLs enable inference of regulatory networks.

An example is the cis-eQTL (rs968567) effect on the lipid-biosynthesis-related gene FADS2 that is modified by expression of the sterol regulatory element-binding transcription factor gene $\operatorname{SREBF} 2(P=4.1 \times$ $10^{-14}, P$ value in Geuvadis $\left.=0.002\right)(\mathbf{F i g} . \mathbf{4 a}, \mathbf{b})$. The eQTL SNP is in close proximity to an SREBF2-binding site (ENCODE ChIP-seq data; Fig. 4c), and it is therefore likely that the SNP modifies the affinity of the FADS2 promoter for SREBF2. SREBF2 showed a significant negative correlation with HDL cholesterol levels (Pearson $r=-0.18$, $\left.P=5.1 \times 10^{-6}\right)$ and a positive correlation with lymphocyte percentage (Pearson $\left.r=0.19, P=1.6 \times 10^{-6}\right)$. Partial correlation analyses showed that the correlation with HDL cholesterol levels was independent of the correlation with lymphocyte percentage (Pearson $r$ on residuals of HDL cholesterol after correcting for lymphocyte percentage $=-0.17$, $P=2.7 \times 10^{-5}$ ), showing that the correlation with HDL cholesterol is not driven by cell type composition. We propose a model where extracellular (HDL) cholesterol levels modify SREBF2 binding to the FADS2 promoter, which in turn has effects on the expression of FADS2 and lipid desaturase activity in the cell. This SNP also increases risk for rheumatoid arthritis, blood metabolite levels and lipid levels; using our method, we now implicate altered binding of SREBF2 as a possible functional mechanism behind these associations.

Another example is a cis-eQTL effect on the MYBL2 gene, encoding a known transcription factor that controls cell division and a tumor suppressor ${ }^{36}$ (Fig. 5a-c). According to ENCODE ChIP-seq data, the top eQTL SNP, rs285205, is located in an EBF1-binding site (Fig. 5d). EBF1 is a known player in B cell differentiation and proliferation. 
a

Correlation matrix of proxy genes affecting the rs285205 (MYBL2) eQTL
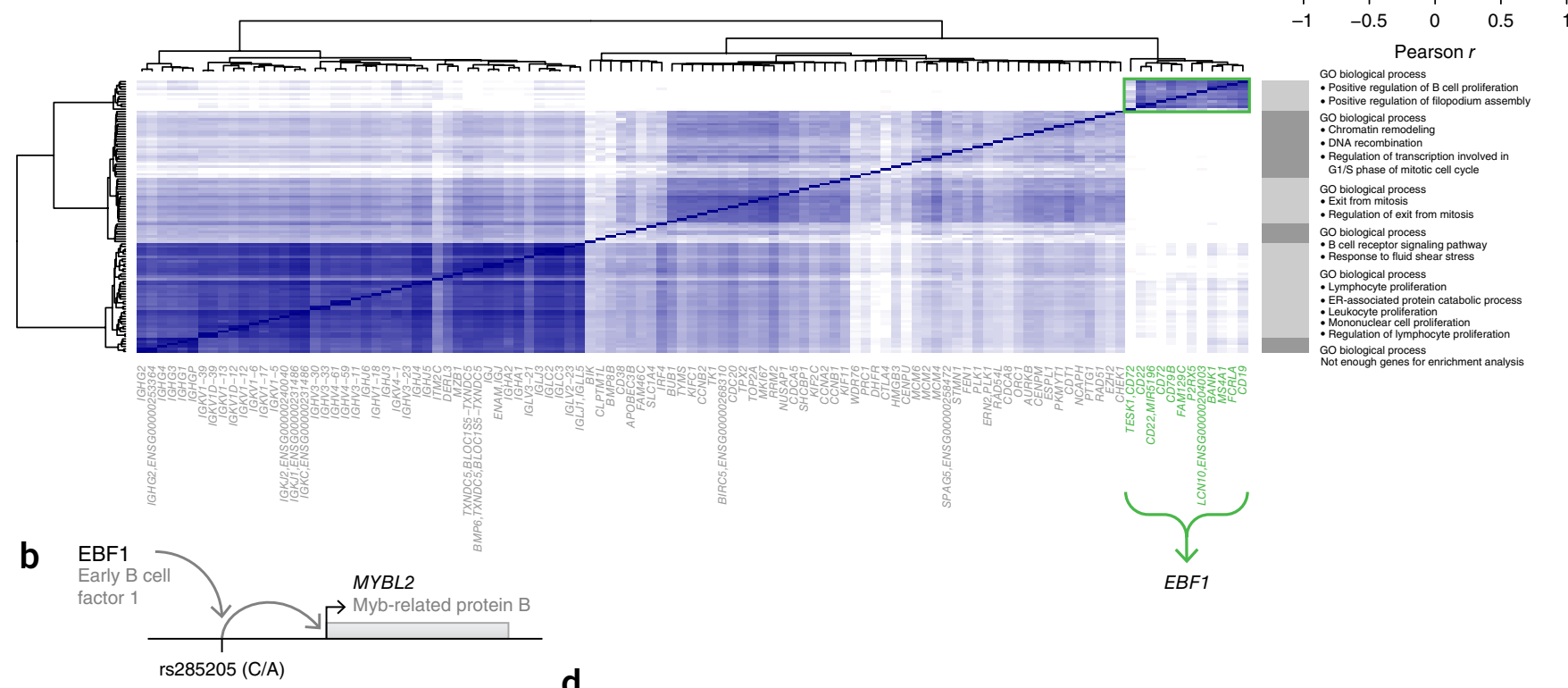

\section{d}

C

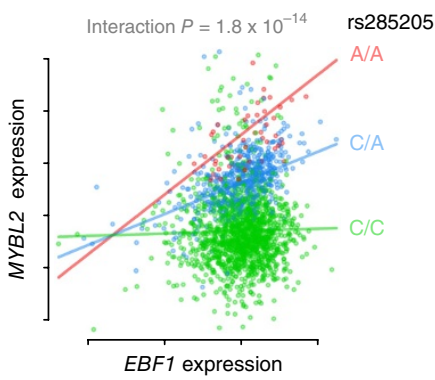

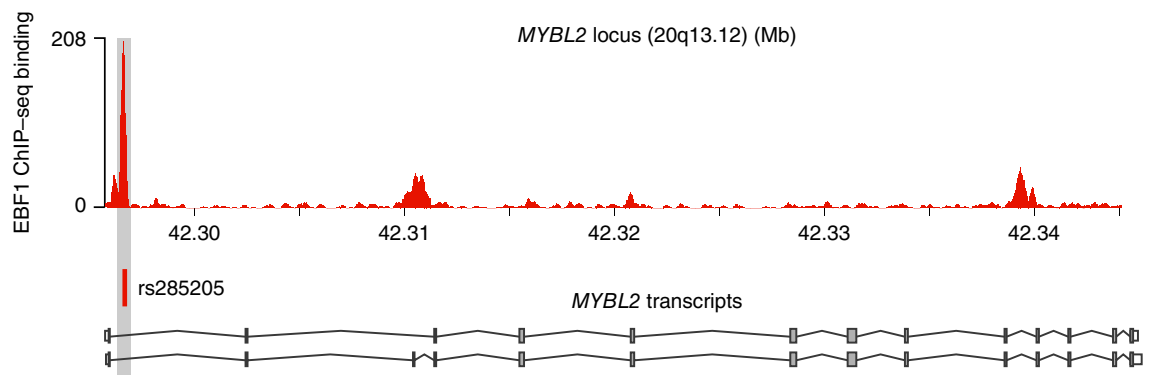

Figure 5 A MYBL2 eQTL is modulated by the B cell proliferation gene EBF1. (a) Heat map of the coexpression of 109 proxy genes that modulate the eQTL effect on MYBL2 expression. Gene functional enrichment analyses on the genes in these clusters show that all are related to proliferation or cell cycle checkpoints. Interestingly, only one cluster increases the magnitude of the MYBL2 eQTL effect, in contrast to the other clusters, which all repress this eQTL. This eQTL-activating cluster is strongly enriched for 'positive regulation of B cell proliferation' $\left(P=1 \times 10^{-7}\right)$, and the strongest proxy gene in this cluster is FCRLA, which is known to be highly expressed in proliferating B cells residing in the germinal center of the lymph nodes (centroblasts) ${ }^{40,41}$. (b) Regulation of MYBL2 by the different cell cycle clusters is likely modulated via EBF1 and rs285205. In our analysis, we had initially only considered genes that were expressed in each of our individuals (Online Methods) and therefore had not studied low-abundance transcription factor genes. When also including these genes, we observed that this cluster of genes is strongly coexpressed with EBF1, a gene encoding a transcription factor that binds at the site of the eQTL SNP, suggesting that EBF1 might drive the eQTL interaction effect for MYBL2. EBF1 is a known player in B cell differentiation and proliferation, and its expression is positively correlated with expression of both $M Y B L 2\left(r=0.11, P=6.99 \times 10^{-7}\right)$ and FCRLA $\left(r=0.8, P \leq 2.2 \times 10^{-16}\right)$. (c) Interaction plot showing that EBF1 expression modifies the eQTL effect of rs285205. The nominal $P$ value is given. (d) ENCODE ChIP-seq data in LCLs show strong binding of EBF1 at rs285205; $n=2,116$ individuals were used in all eQTL analyses.

Although FCRLA expression was the strongest modifier of the eQTL, EBF1 expression was highly correlated with FCRLA expression and showed a significant interaction effect on the MYBL2 eQTL $(P=1.8$ $\times 10^{-14}$ ) (Fig. 5c). The eQTL SNP therefore likely affects the binding affinity of EBF1.

In conclusion, we greatly expanded the catalog of SNPs that have a known regulatory function. To gain a better understanding of the biology behind these regulatory variants, we assessed the context dependency of the eQTLs and determined 2,743 to be context dependent. With future increases in sample size, we expect that it will become possible to identify more unanticipated intrinsic factors and external stimuli that modify the downstream effects of genetic risk factors. As such, our approach complements perturbation experiments in gaining better insight into regulatory networks and their stimuli, and it can easily be applied to other tissues. A caveat of our hypothesisfree approach is that it is not always straightforward to understand which internal or external cues the proxy genes represent. Integration with other expression or transcription factor binding data, as we have done here, is therefore instrumental for the interpretation of context-dependent eQTLs.

\section{METHODS}

Methods, including statements of data availability and any associated accession codes and references, are available in the online version of the paper.

Note: Any Supplementary Information and Source Data files are available in the online version of the paper.

\section{ACKNOWLEDGMENTS}

This work was performed within the framework of the Biobank-Based Integrative Omics Studies (BIOS) consortium funded by BBMRI-NL, a research infrastructure financed by the Dutch government (NWO 184.021.007). Samples were contributed 
by LifeLines (http://lifelines.nl/lifelines-research/general), the Leiden Longevity Study (http://www.healthy-ageing.nl/ and http://www.leidenlangleven.nl/), the Rotterdam Studies (http://www.erasmus-epidemiology.nl/research/ergo.htm) and the CODAM study (http://www.carimmaastricht.nl/). We thank the participants of all aforementioned biobanks and acknowledge the contributions of the investigators to this study (Supplementary Note). This work was carried out on the Dutch national e-infrastructure with the support of SURF Cooperative and the Groningen Center for Information Technology (G.J.C. Strikwerda, W. Albers, R. Teeninga, H. Gankema and H. Wind) and Target storage (E. Valentyn and R. Williams). Target is supported by Samenwerkingsverband Noord Nederland, the European Fund for Regional Development, the Dutch Ministry of Economic Affairs, Pieken in de Delta, and the provinces of Groningen and Drenthe. This work is supported by a grant from the European Research Council (ERC Starting Grant agreement 637640 ImmRisk) to L.F. The Rotterdam Study is funded by Erasmus Medical Center and Erasmus University, Rotterdam, the Netherlands Organization for Health Research and Development (ZonMw), the Research Institute for Diseases in the Elderly (RIDE), the Ministry of Education, Culture and Science, the Ministry for Health, Welfare and Sports, the European Commission (DG XII) and the municipality of Rotterdam. The authors are grateful to the study participants, the staff from the Rotterdam Study, and the participating general practitioners and pharmacists. The generation and management of GWAS genotype data for the Rotterdam Study are supported by the Netherlands Organization for Scientific Research NWO Investments (175.010.2005.011, 91103-012). This study is funded by the Research Institute for Diseases in the Elderly (014-93-015; RIDE2) and Netherlands Genomics Initiative (NGI)/Netherlands Organization for Scientific Research (NWO) project 050-060-810. We thank P. Arp, M. Jhamai, M. Verkerk, L. Herrera and M. Peters for their help in creating the GWAS database. Work on cell count estimation was funded by NWO 863.13.011. The LifeLines Deep cohort is made possible by grants from the Top Institute of Food and Nutrition (TiFN GH0001), an ERC advanced grant (FP/2007-2013/ERC grant 2012322698) and a Spinoza prize (NWO SPI 92-266) to C.W.

\section{AUTHOR CONTRIBUTIONS}

B.T.H., P.A.C.t.H., J.B.J.v.M., A.I., R.J. and L.F. formed the management team of the BIOS consortium. D.I.B., R.P., J.v.D., J.J.H., M.M.J.V.G., C.D.A.S., C.J.H.v.d.K., C.G.S., C.W., L.F., A.Z., E.F.T., P.E.S., M.B., J.D., D.v.H., J.H.V., L.H.v.d.B., C.M.v.D., A.H., A.I. and A.G.U. managed and organized the biobanks. J.B.J.v.M., P.M.J., M. Verkerk and J.v.R. generated RNA-seq data. H.M., M.v.I., M.v.G., W.A., J.B., D.V.Z., R.J., P.v.t.H., P.D., M. Verkerk, M. Vermaat, I.N., M.A.S., P.A.C.t.H., B.T.H. and M.M. were responsible for data management and the computational infrastructure. D.V.Z., P.D., M. Vermaat, M.v.I., F.v.D., M.v.G., W.A., M.J.B., N.d.K., H.-J.W., S.M.K., Y.L., M.A.S., P.A.C.t.H. and L.F. performed the data analysis. D.V.Z., P.D., P.A.C.t.H. and L.F. drafted the manuscript.

\section{COMPETING FINANCIAL INTERESTS}

The authors declare no competing financial interests.

Reprints and permissions information is available online at http://www.nature.com/ reprints/index.html.

1. Schaub, M.A., Boyle, A.P., Kundaje, A., Batzoglou, S. \& Snyder, M. Linking disease associations with regulatory information in the human genome. Genome Res. 22, 1748-1759 (2012).

2. Hindorff, L.A. et al. Potential etiologic and functional implications of genome-wide association loci for human diseases and traits. Proc. Natl. Acad. Sci. USA 106, 9362-9367 (2009).

3. Musunuru, K. et al. From noncoding variant to phenotype via SORT1 at the $1 \mathrm{p} 13$ cholesterol locus. Nature 466, 714-719 (2010).

4. Cvejic, A. et al. SMIM1 underlies the Vel blood group and influences red blood cell traits. Nat. Genet. 45, 542-545 (2013).

5. Smemo, S. et al. Obesity-associated variants within $F T O$ form long-range functional connections with IRX3. Nature 507, 371-375 (2014).

6. Claussnitzer, M. et al. FTO obesity variant circuitry and adipocyte browning in humans. N. Engl. J. Med. 373, 895-907 (2015).

7. Fu, J. et al. Unraveling the regulatory mechanisms underlying tissue-dependent genetic variation of gene expression. PLoS Genet. 8, e1002431 (2012).

8. Fairfax, B.P. et al. Genetics of gene expression in primary immune cells identifies cell type-specific master regulators and roles of HLA alleles. Nat. Genet. 44, 502-510 (2012)

9. Andiappan, A.K. et al. Genome-wide analysis of the genetic regulation of gene expression in human neutrophils. Nat. Commun. 6, 7971 (2015).
10. Francesconi, M. \& Lehner, B. The effects of genetic variation on gene expression dynamics during development. Nature 505, 208-211 (2014).

11. Powell, J.E. et al. Genetic control of gene expression in whole blood and lymphoblastoid cell lines is largely independent. Genome Res. 22, 456-466 (2012).

12. Deelen, P. et al. Calling genotypes from public RNA-sequencing data enables identification of genetic variants that affect gene-expression levels. Genome Med. 7, 30 (2015)

13. Westra, H.-J. et al. Cell specific eQTL analysis without sorting cells. PLoS Genet. 11, e1005223 (2015).

14. Fairfax, B.P. et al. Innate immune activity conditions the effect of regulatory variants upon monocyte gene expression. Science 343, 1246949 (2014).

15. Çalışkan, M., Baker, S.W., Gilad, Y. \& Ober, C. Host genetic variation influences gene expression response to rhinovirus infection. PLoS Genet. 11, e1005111 (2015).

16. Lee, M.N. et al. Common genetic variants modulate pathogen-sensing responses in human dendritic cells. Science 343, 1246980 (2014).

17. Barreiro, L.B. et al. Deciphering the genetic architecture of variation in the immune response to Mycobacterium tuberculosis infection. Proc. Natl. Acad. Sci. USA 109, 1204-1209 (2012).

18. van Greevenbroek, M.M.J. et al. The cross-sectional association between insulin resistance and circulating complement $\mathrm{C} 3$ is partly explained by plasma alanine aminotransferase, independent of central obesity and general inflammation (the CODAM study). Eur. J. Clin. Invest. 41, 372-379 (2011).

19. Tigchelaar, E.F. et al. Cohort profile: LifeLines DEEP, a prospective, genera population cohort study in the northern Netherlands: study design and baseline characteristics. BMJ Open 5, e006772 (2015).

20. Schoenmaker, M. et al. Evidence of genetic enrichment for exceptional survival using a family approach: the Leiden Longevity Study. Eur. J. Hum. Genet. 14, 79-84 (2006).

21. Hofman, A. et al. The Rotterdam Study: 2014 objectives and design update. Eur. J. Epidemiol. 28, 889-926 (2013).

22. Westra, H.-J. et al. Systematic identification of trans eQTLs as putative drivers of known disease associations. Nat. Genet. 45, 1238-1243 (2013).

23. Lappalainen, T. et al. Transcriptome and genome sequencing uncovers functiona variation in humans. Nature 501, 506-511 (2013).

24. Wood, A.R. et al. Allelic heterogeneity and more detailed analyses of known loc explain additional phenotypic variation and reveal complex patterns of association. Hum. Mol. Genet. 20, 4082-4092 (2011).

25. Ritchie, G.R.S., Dunham, I., Zeggini, E. \& Flicek, P. Functional annotation of noncoding sequence variants. Nat. Methods 11, 294-296 (2014).

26. Andersson, R. et al. An atlas of active enhancers across human cell types and tissues. Nature 507, 455-461 (2014).

27. Naranbhai, V. et al. Genomic modulators of gene expression in human neutrophils, Nat. Commun. 6, 7545 (2015).

28. Raj, T. et al. Polarization of the effects of autoimmune and neurodegenerative risk alleles in leukocytes. Science 344, 519-523 (2014).

29. Idaghdour, Y. et al. Geographical genomics of human leukocyte gene expression variation in southern Morocco. Nat. Genet. 42, 62-67 (2010).

30. Yao, C. et al. Sex- and age-interacting eQTLs in human complex diseases. Hum. Mol. Genet. 23, 1947-1956 (2014).

31. Adams, D. et al. BLUEPRINT to decode the epigenetic signature written in blood. Nat. Biotechnol. 30, 224-226 (2012)

32. Doré, L.C. \& Crispino, J.D. Transcription factor networks in erythroid cell and megakaryocyte development. Blood 118, 231-239 (2011)

33. Hall, M.A. et al. The critical regulator of embryonic hematopoiesis, SCL, is vital in the adult for megakaryopoiesis, erythropoiesis, and lineage choice in CFU-S12. Proc. Natl. Acad. Sci. USA 100, 992-997 (2003).

34. Pevny, L. et al. Erythroid differentiation in chimaeric mice blocked by a targeted mutation in the gene for transcription factor GATA-1. Nature 349, 257-260 (1991).

35. Rusinova, I. et al. Interferome v2.0: an updated database of annotated interferonregulated genes. Nucleic Acids Res. 41, D1040-D1046 (2013).

36. Heinrichs, S. et al. MYBL2 is a sub-haploinsufficient tumor suppressor gene in myeloid malignancy. eLife 2, e00825 (2013).

37. Platanias, L.C. Mechanisms of type-I- and type-II-interferon-mediated signalling. Nat. Rev. Immunol. 5, 375-386 (2005).

38. Ivashkiv, L.B. \& Donlin, L.T. Regulation of type I interferon responses. Nat. Rev. Immunol. 14, 36-49 (2014).

39. McLeay, R.C. \& Bailey, T.L. Motif Enrichment Analysis: a unified framework and an evaluation on ChIP data. BMC Bioinformatics 11, 165 (2010).

40. Facchetti, F., Cella, M., Festa, S., Fremont, D.H. \& Colonna, M. An unusual Fc receptor-related protein expressed in human centroblasts. Proc. Natl. Acad. Sci. USA 99, 3776-3781 (2002).

41. Rosén, A. et al. Lymphoblastoid cell line with B1 cell characteristics established from a chronic lymphocytic leukemia clone by in vitro EBV infection. Oncolmmunology 1, 18-27 (2012) 


\section{ONLINE METHODS}

Cohort descriptions. The four cohorts used in our BIOS study are briefly described below. The age range of the individuals differed for the different biobanks (Supplementary Fig. 7). The number of samples per cohort used in our study can be found in Supplementary Table 1.

CODAM. The Cohort on Diabetes and Atherosclerosis Maastricht $(\mathrm{CODAM})^{18}$ consists of a selection of 547 subjects from a larger populationbased cohort ${ }^{42}$. Inclusion of subjects into CODAM was based on a moderately increased risk of developing cardiometabolic diseases such as type 2 diabetes and/or cardiovascular disease. Subjects were included if they were of European descent, over 40 years of age and additionally met at least one of the following criteria: increased body mass index (BMI; $>25$ ), a positive family history of type 2 diabetes, a history of gestational diabetes and/or glycosuria, or use of antihypertensive medication.

$L L D$. The LifeLines-DEEP (LLD) cohort $^{19}$ is a subcohort of the LifeLines cohort $^{43}$ with additional molecular data on 1,500 participants. LifeLines is a multidisciplinary prospective population-based cohort study examining the health and health-related behaviors of 167,729 individuals living in the northern parts of the Netherlands using a unique three-generation design. It employs a broad range of investigative procedures assessing the biomedical, sociodemographic, behavioral, physical and psychological factors contributing to health and disease in the general population, with a special focus on multi-morbidity and complex genetics.

LLS. The aim of the Leiden Longevity Study (LLS) ${ }^{20}$ is to identify genetic factors influencing longevity and examine their interaction with the environment to develop interventions by which to increase health at older ages. To this end, long-lived siblings of European descent were recruited together with their offspring and their offspring's partners, on the condition that at least two long-lived siblings were alive at the time of ascertainment. For men, the age criterion was 89 years or older; for women, the age criterion was 91 years or older. These criteria led to the ascertainment of 944 long-lived siblings from 421 families, together with 1,671 of their offspring and 744 partners.

$R S$. The Rotterdam Study ${ }^{21}$ is a single-center, prospective population-based cohort study conducted in Rotterdam, the Netherlands. Subjects were included in different phases from the start of the study in 1998, with a total of 14,926 men and women aged 45 years and over included as of late 2008. The main objective of the Rotterdam Study is to investigate the prevalence and incidence of and risk factors for chronic diseases to contribute to better prevention and treatment of such diseases in the elderly.

Ethical approval. The ethical approval for this study lies with the individual participating cohorts (CODAM, LLD, LLS and RS) ${ }^{18-21}$.

RNA data preparation and sequencing. Total RNA from whole blood was depleted of globin transcripts using the Ambion GLOBINclear kit and subsequently processed for sequencing using the Illumina TruSeq version 2 library preparation kit. Paired-end sequencing of $2 \times 50$-bp reads was performed using the Illumina HiSeq 2000 platform, pooling ten samples per lane and aiming for $>15$ million read pairs per sample. Finally, the read sets were generated for each sample using CASAVA, retaining only reads passing the Illumina Chastity Filter for further processing.

Preprocessing. The quality of the raw reads was checked using FastQC (http://www.bioinformatics.babraham.ac.uk/projects/fastqc/). The adaptors identified by FastQC (v0.10.1) were clipped using cutadapt (v1.1), applying default settings (min overlap 3, min length 25). Sickle (v1.200) (https://github. $\mathrm{com} /$ najoshi/sickle) was used to trim low-quality ends from the reads (min length 25 , min quality 20 ).

Alignment. Read alignment was performed using STAR 2.3.0e (ref. 44). To avoid reference mapping bias, all Genome of the Netherlands (GoNL) SNPs with minor allele frequency (MAF) $>0.01$ in the reference genome were masked as N's (ref. 45). Read pairs with at most eight mismatches, mapping to at most five positions, were used.

Alignment statistics. Mapping statistics from the BAM files were acquired through SAMtools flagstat (v0.1.19-44428cd). The $5^{\prime}$ and $3^{\prime}$ coverage bias, duplication rate and insert sizes were assessed using Picard tools (v1.86).

Expression quantification. We estimated expression on the gene, exon, exon ratio and poly(A) ratio levels using Ensembl v.71 annotation (which corresponds to GENCODE v.16).
Overlapping exons (on either of the two strands) were merged into metaexons, and expression was quantified for the whole meta-exon. Custom scripts were developed for this purpose that use coverage per base from coverageBed and intersectBed from the BEDtools suite (v2.17.0) ${ }^{46}$ and R (v2.15.1). This resulted in base counts per exon or meta-exon.

Gene expression, as base count per gene, was calculated as the sum of the expression values for all exons of each gene (excluding meta-exons). Overlapping gene parts were counted separately from unique gene parts throughout this manuscript.

Expression of exons relative to their gene (exon ratio) was calculated by dividing the exon base counts by the summed base counts for all exons of the same gene. Meta-exons overlapping with multiple genes were discarded.

Overlapping 3' UTRs for the same gene, as annotated in Ensembl, were merged by gene. A collection of poly(A) sites was retrieved from PolyA_DB, and the annotated $3^{\prime}$ ends of transcripts were obtained from Ensembl. These poly(A) sites were used to split the merged $3^{\prime}$ UTRs into bins. To avoid small bins, which tend to give noisy ratios, we applied some filtering to the poly $(\mathrm{A})$ sites. Poly(A) sites located no more than $10 \mathrm{bp}$ from the start or from the end of the $3^{\prime}$ UTR were discarded. Additionally, sites that were no more than $10 \mathrm{bp}$ apart were merged (if the number of sites was even, the first site downstream was used). For all genes with at least two bins (corresponding to at least two potential poly(A) sites), we calculated the ratio of base counts for every two neighboring bins (poly(A) ratio).

Genotype data. Data generation. Genotype data were generated for each cohort individually. Details on the methods used can be found in the individual papers $\left(\mathrm{CODAM}^{42}, \mathrm{LLD}^{19}, \mathrm{LLS}^{47}\right.$ and $\left.\mathrm{RS}^{21}\right)$.

Imputation and quality control. The genotype data were harmonized to GoNL ${ }^{48}$ using Genotype Harmonizer ${ }^{49}$ and were subsequently imputed per cohort with IMPUTE2 (ref. 50) using the GoNL reference panel ${ }^{51}$ (v5). Quality control was also performed per cohort. We removed SNPs with an imputation info score below 0.5, a Hardy-Weinberg equilibrium $P$ value smaller than $1 \times 10^{-4}$, a call rate below $95 \%$ or a MAF smaller than 0.05 . In total, $9,333,740$ SNPs passed quality control in at least one data set.

Quality control. To identify low-quality samples, we applied several quality metrics and used a combination of them to decide whether to exclude a sample from further analyses.

Read counts. For each sample, the total number of mapped reads was used as a quality measure. Samples for which these counts were less than $70 \%$ were flagged and excluded from the analysis.

Exon and gene expression correlation. For each pair of samples, the Spearman correlation of their expression was calculated on the gene and exon levels. From these values, the median Spearman correlation for each sample was calculated ( $D$ statistic). Samples with $D$ statistics lower than 0.85 were excluded from the analysis.

Genotype concordance. As an extra quality control step, we compared imputed genotypes to those derived from RNA-seq. Concordance is expected to be low in cases of poor-quality RNA-seq or imputed genotype data or in cases of sample mix-up.

RNA-seq genotypes were called using SAMtools mpileup ${ }^{52}$ (with the following parameters: -A -B -Q 0 -s -d10000000; calling only GoNL SNPs with MAF $>0.01$ ) and SNVMix2 (ref. 53). Only genotypes with posterior probabilities higher than 0.8 were included. We determined the genotype concordance per sample as the genotype correlation of high-confidence SNPs (SNPs with a mean genotype correlation across all samples of no lower than 0.9 ). Outlier samples, for which the genotype concordance was less than 0.9 , were flagged and excluded from the analysis.

Heterozygosity rate. A maximum heterozygosity rate of 0.52 was used to exclude contaminated RNA-seq samples. This rate was calculated using the same high-quality genotypes used for the genotype concordance calculations.

Mix-up mapping. Previously, we showed that sample mix-ups occur frequently in genomics data sets, introducing noise into subsequent analyses ${ }^{54}$. We checked the data for mix-ups using this published method and flagged possibly mixed samples.

QTL mapping. We used our previously described pipeline ${ }^{22}$ to perform eQTL mapping. We mapped QTLs using Spearman rank correlation on imputed 
genotype dosages in each cohort and then ran a meta-analysis combining the results by weighted $z$-score method. To control the FDR at 0.05 , we created a null distribution by permuting sample labels for the expression data, repeating this process ten times.

Expression data normalization. Expression data on the gene and exon levels were first normalized using trimmed mean of $M$ (TMM) values ${ }^{55}$. Expression values were then $\log _{2}$ transformed, probe and sample means were centered to zero, and their standard deviation was scaled to one. To correct for batch effects, principal-component analysis (PCA) was run on the sample Spearman correlation matrix and the first 25 principal components were removed ${ }^{22}$. We observed that removing these principal components resulted in detection of the highest number of eQTLs. To verify that none of these 25 principal components were under genetic control, we ran separate QTL mapping on each principal component and ensured that there were no SNPs associated with them.

Exon ratio and poly(A) ratio expression data were not normalized, as ratios are not dependent on library size and we used non-parametric statistics.

Cis-QTL mapping. To run cis-QTL mapping, we tested genes (or exons, exon ratios or poly(A) ratios) and SNPs located within $250 \mathrm{~kb}$ of a gene (or exon) center. Only SNPs with MAF $\geq 0.05$, call rate $\geq 0.95$ and Hardy-Weinberg equilibrium $P$ value $\geq 0.001$ were included. We identified independent QTL effects by stepwise regression: we found secondary QTLs by regressing out the primary QTLs and identified tertiary QTLs by regressing out primary and secondary QTLs. This procedure was repeated until no more independent effects were found. We acknowledge that it might be possible that some of the identified independent effects might actually tag untyped variants.

Set of background SNPs for functional enrichment analyses. To assess the functional enrichment of expression SNPs (eSNPs) for each QTL, we created a list of background SNPs that we compared to the real set. For each eQTL SNP, we selected the variants within a 50,000-bp window with a MAF differing by no more than 0.05 from that of the eQTL SNP and LD $r^{2} \leq 0.5$. From the variants that met these criteria, we selected the one that was physically closest to the eQTL SNP as the background SNP.

Replication of $\boldsymbol{c i s}$-eQTLs. The first replication data set comprised Geuvadis RNA-seq data from LCLs ${ }^{23}$. For replication, we obtained raw RNA-seq reads from 373 European samples and processed them using the same alignment and quality control pipeline as we used on the BIOS data. For eQTL mapping, we regressed out the first 20 principal components from the expression data (because of the smaller sample size of the Geuvadis data set). To replicate BIOS eQTLs in Geuvadis, we took all significant eQTLs (the top SNP for each gene) from BIOS and ran eQTL mapping in Geuvadis, testing only these eQTLs. We then checked how many eQTLs out of all those tested were replicated and for how many of the replicated eQTLs the allelic direction was opposite. We performed the same analysis in the other direction, testing how many of the Geuvadis eQTLs were replicated in the BIOS data.

The second data set for replication comprised a meta-analysis of 5,311 peripheral blood samples analyzed by microarray ${ }^{22}$. As raw data were not available for this data set, we used all significant eQTLs (FDR $<0.05)$ identified in the meta-analysis, mapped the microarray probes to genes and exons using Ensembl v71 gene annotation, and then tested these SNP-gene and SNP-exon combinations in the BIOS data.

GWAS annotation. To annotate eQTLs with known disease or trait associations, we used a set of 6,321 SNPs derived from the NHGRI GWAS catalog and a set of reported Immunochip associations, each with reported $P \leq 5 \times 10^{-8}$ (Supplementary Table 6).

Interaction analysis. For an overview of the method used for the interaction analysis, see Supplementary Figure 2. The interaction analysis was performed using the following linear model

$$
Y \approx I+\beta_{1} G+\beta_{2} P+\beta_{3} P \times G
$$

where $Y$ is the eQTL gene expression, $G$ is the eQTL SNP genotype, $P$ is the proxy gene, $P \times G$ is the interaction term between the proxy gene and the genotype, $I$ is the intercept, and $\beta_{1}, \beta_{2}$ and $\beta_{3}$ are regression coefficients.

As a linear model is parametric and thus more sensitive to outliers and nonnormal distributions than our non-parametric eQTL model, we performed stricter quality control. We found that several metrics introduced outliers in our data that confounded the linear regression analyses. These metrics were the percentage of coding bases, the median $3^{\prime}$ bias, the percentage of uniquely mapped reads and the percentage of mRNA bases (Supplementary Fig. 8). On the basis of these metrics, we removed 75 samples and used the remaining 2,041 samples in the interaction analyses. We confined the interaction analysis to genes with at least one mapped read in all samples; this criterion was used for both the proxy genes and the eQTL genes. As a result, we tested 29,750 genes as potential proxies and 17,291 eQTL effects.

Normalization for the expression of eQTL genes is different from that for the expression of proxy genes. The gene expression data for eQTL genes were corrected using covariates for the source biobank, the first 25 principal components, sex, the median $3^{\prime}$ bias, the median $5^{\prime}$ bias, GC content and the percentage of intronic bases. To detect biologically meaningful interaction effects, we also regressed out the interaction effects for sex, the median $3^{\prime}$ bias, the median $5^{\prime}$ bias, GC content and the percentage of intronic bases. The expression data used in the interaction term were processed in a similar manner, with the exception that we did not correct for principal components, as this would have removed correlations with cell type, and we did not correct for interactions with technical covariates.

We excluded interactions where the eQTL SNP showed a significant eQTL effect on the tested proxy gene, as we wanted to exclude cases in which the gene giving the interaction effect was in the same locus as the tested eQTL gene.

We then performed an iterative interaction analysis by regressing the top covariate in a stepwise manner. After the first round of interaction analysis, we identified the covariate having the highest chi2sum

$$
\sum_{e \in E} z_{e}^{2}
$$

where $e$ is an eQTL from the set of all eQTLs $(E)$ and $z_{e}^{2}$ is the squared interaction $z$ score of the current covariate with the eQTL $e$, over all interaction $z$ scores. We regressed out this covariate from the covariate and gene expression data and repeated the interaction analysis. This procedure was repeated ten times. For each top covariate, we identified a set of covariates (module) with a similar interaction pattern by taking the top 100 covariates having the highest chi2sum difference between the current interaction analysis step and the previous step (effectively identifying coexpressed genes). These covariates were mostly highly coexpressed with the top covariate in the module (Fig. 2c).

To determine the significance level of interactions, we permuted genotype sample labels and ran the interaction analysis. This enabled us to determine which eQTLs significantly interacted with the top covariate of the module with FDR $\leq 0.05$.

We ran interaction analysis at the exon and exon ratio levels in a similar manner as for the gene level. The implementation and manual for our method can be found at https://github.com/molgenis/systemsgenetics/wiki/Discoveryof-hidden-confounders-of-QTLs.

Interaction module functions. To find the prevalent cell type for each module, we used several sources of information. Some of the BIOS biobanks had cell counts available, making it possible to correlate the top 100 covariates of each module with cell type percentages.

As an additional source of evidence, we used expression profiles for isolated populations of 17 of the major cell types in blood generated by the BLUEPRINT consortium ${ }^{31}$.

To determine the putative function of each module, we performed pathway enrichment analysis using GeneNetwork ${ }^{56,57}$ on the top 100 covariates in the module and on all eQTL genes having a significant interaction with the top covariate of the module.

To gain more insight into the function of the modules we identified, we overlapped the interaction results with those from several previous studies of stimulated cells and response QTLs (reQTLs), including a study of peripheral blood mononuclear cells infected with rhinovirus ${ }^{15}$ and a study of monocytes 
treated with LPS (collected at 2 and $24 \mathrm{~h}$ after treatment) and interferon ${ }^{14}$. To investigate whether each interaction module represented an antiviral or antibacterial response, we checked for enrichment of the differentially expressed genes reported for each stimulation (with $-1<\log$ (fold change) $<1$ ) within the top 100 covariates for each interaction module by performing a one-tailed Fisher's exact test to determine the significance. We also determined whether the reported reQTLs showed significantly stronger interaction with the top covariate of each module by performing a Wilcoxon rank-sum test on interaction $z$ scores.

We checked whether there was enrichment of binding for particular transcription factors using ChIP-seq data from $\mathrm{ENCODE}^{58}$. First, we determined which transcription factors overlapped with the eQTL SNP or a variant in very strong LD $\left(r^{2} \geq 0.99\right)$. Then, using a Fisher's exact test, we determined whether there was any enrichment in overlap between the genes assigned to a module and the genes not significantly assigned to this module.

Using interaction modules to better understand disease mechanisms. We extracted the genes regulated by any type of top QTL variant in strong LD $\left(r^{2} \geq 0.8\right)$ with top GWAS hits. Coexpression was assessed for these genes in our data, and Cytoscape 3.2.1 (ref. 59) was used to create network plots. Assignment to specific clusters was performed using the $\mathrm{R}$ implementation of Affinity Propagation ${ }^{60,61}$. Cell-type-specific expression levels were based on the RNA-seq data generated by the BLUEPRINT consortium ${ }^{31}$ and were plotted using gplots. We performed gene function enrichment analysis using GeneNetwork ${ }^{56}$.

Cell-type-specific eQTL mapping. Cell-type-specific eQTLs were identified using the same method we used for the gene-based interaction analyses. However, here we used cell type percentages instead of the expression of other genes. As not all cohorts measured cell counts, we estimated counts for cohorts without this information. RNA-seq and cell count data for 628 samples from the LLD cohort and 650 samples from the LLS cohort were used to build prediction models for cell count using an in-house predictor for neutrophils, lymphocytes, monocytes, eosinophils and basophils. We evaluated this method using cross-validation (Supplementary Fig. 9). The models were applied to RNA-seq data for 185 samples from the CODAM cohort and 14 samples from the LLS cohort to predict cell counts for the five cell types. In addition, the prediction models were applied to estimate cell counts for neutrophils, eosinophils and basophils, using RNA-seq data from 652 samples from the RS cohort in which cell counts for lymphocytes and monocytes were available.

BLUEPRINT tissue-specific expression data analysis. BLUEPRINT data was downloaded from their ftp site (ftp://ftp.ebi.ac.uk/pub/databases/blueprint). All RNA-seq data from venous blood, myeloid cell and erythroblast samples were downloaded. Read counts were obtained according to the gene quantification performed by the Center for Genomic Regulation. Subsequently, TMM normalization ${ }^{55}$ was performed. Averaged normalized log-transformed counts per million per cell type were used to draw heat maps. For each module, we extracted corresponding genes on the basis of their Ensembl gene identifiers (for meta-exons, we used the first Ensembl identifier; three noncoding RNAs could not be extracted from the BLUEPRINT data). Furthermore, the $\mathrm{R}$ package pheatmap (1.0.7) was used to generate heat maps.
Data availability. Raw RNA-seq data can be obtained from the European Genome-phenome Archive (EGA; accession EGAD00001001623). Genotype data are available from the respective biobanks: LLS (http://www. leidenlangleven.nl/en/home; e-mail: m.beekman@lumc.nl), LifeLines (https:// lifelines.nl/lifelines-research/access-to-lifelines; e-mail: 1lscience@umcg.nl), CODAM (e-mail: m.vangreevenbroek@maastrichtuniversity.nl) and RS (http://www.erasmusmc.nl/epi/research/The-Rotterdam-Study/?lang=en; e-mail: m.a.ikram@erasmusmc.nl).eQTL results can be accessed via our web browser (http://genenetwork.nl/biosqtlbrowser/).

42. van Dam, R.M., Boer, J.M., Feskens, E.J.M. \& Seidell, J.C. Parental history of diabetes modifies the association between abdominal adiposity and hyperglycemia. Diabetes Care 24, 1454-1459 (2001).

43. Scholtens, S. et al. Cohort profile: LifeLines, a three-generation cohort study and biobank. Int. J. Epidemiol. 44, 1172-1180 (2015).

44. Dobin, A. et al. STAR: ultrafast universal RNA-seq aligner. Bioinformatics 29, 15-21 (2013).

45. Liu, Z. et al. Comparing computational methods for identification of allele-specific expression based on next generation sequencing data. Genet. Epidemiol. 38 591-598 (2014).

46. Quinlan, A.R. \& Hall, I.M. BEDTools: a flexible suite of utilities for comparing genomic features. Bioinformatics 26, 841-842 (2010).

47. Deelen, J. et al. Genome-wide association meta-analysis of human longevity identifies a novel locus conferring survival beyond 90 years of age. Hum. Mol. Genet. 23, 4420-4432 (2014).

48. Genome of the Netherlands Consortium. Whole-genome sequence variation, population structure and demographic history of the Dutch population. Nat. Genet. 46, 818-825 (2014).

49. Deelen, P. et al. Genotype harmonizer: automatic strand alignment and format conversion for genotype data integration. BMC Res. Notes 7, 901 (2014).

50. Howie, B.N., Donnelly, P. \& Marchini, J. A flexible and accurate genotype imputation method for the next generation of genome-wide association studies. PLoS Genet 5, e1000529 (2009).

51. Deelen, P. et al. Improved imputation quality of low-frequency and rare variants in European samples using the 'Genome of The Netherlands'. Eur. J. Hum. Genet. 22, 1321-1326 (2014).

52. Li, H. et al. The Sequence Alignment/Map format and SAMtools. Bioinformatics 25, 2078-2079 (2009).

53. Goya, R. et al. SNVMix: predicting single nucleotide variants from next-generation sequencing of tumors. Bioinformatics 26, 730-736 (2010).

54. Westra, H.-J. et al. MixupMapper: correcting sample mix-ups in genome-wide datasets increases power to detect small genetic effects. Bioinformatics 27 2104-2111 (2011).

55. Robinson, M.D. \& Oshlack, A. A scaling normalization method for differential expression analysis of RNA-seq data. Genome Biol. 11, R25 (2010).

56. Fehrmann, R.S.N. et al. Gene expression analysis identifies global gene dosage sensitivity in cancer. Nat. Genet. 47, 115-125 (2015).

57. Pers, T.H. et al. Biological interpretation of genome-wide association studies using predicted gene functions. Nat. Commun. 6, 5890 (2015).

58. Landt, S.G. et al. ChIP-seq guidelines and practices of the ENCODE and modENCODE consortia. Genome Res. 22, 1813-1831 (2012).

59. Cline, M.S. et al. Integration of biological networks and gene expression data using Cytoscape. Nat. Protoc. 2, 2366-2382 (2007).

60. Frey, B.J. \& Dueck, D. Clustering by passing messages between data points. Science 315, 972-976 (2007).

61. Bodenhofer, U., Kothmeier, A. \& Hochreiter, S. APCluster: an R package for affinity propagation clustering. Bioinformatics 27, 2463-2464 (2011). 